\title{
Validation of the Estimation of Corneal Aberrations From Videokeratography in Keratoconus
}

\author{
Sergio Barbero, BSc; Susana Marcos, PhD; Jesus Merayo-Lloves, MD, PhD; \\ Esther Moreno-Barriuso, PhD
}

\begin{abstract}
PURPOSE : To validate the estimation of corneal aberrations from videokeratography against a laser ray tracing technique that measured total eye aberrations, in eyes without keratoconus (ie, cornea-dominated wave aberrations).

METHODS: We measured total and corneal wave aberrations of three eyes diagnosed with keratoconus by slit-lamp microscopy and corneal topography: two eyes from one patient with early keratoconus and one eye with more advanced keratoconus. Total aberrations were measured with laser ray tracing. Corneal aberrations were obtained from corneal elevation data measured with a corneal videokeratoscope using custom software that performs virtual ray tracing on the measured front corneal surface.

RESULTS: The keratoconus eyes showed a dramatic increase in aberrations (both corneal and total) particularly coma-like terms, which were 3.74 times higher on average than normal. Anterior corneal surface aberrations and total aberrations were similar in keratoconus. This similarity was greater for the early keratoconus patient, suggesting a possible implication of the posterior corneal surface in advanced keratoconus.
\end{abstract}

From the Instituto de Óptica, Consejo Superior de Investigaciones Científicas, Madrid, Spain (Barbero, Marcos, Moreno-Barriuso), Instituto de Oftalmobiología Aplicada, Universidad de Valladolid, Spain (MerayoLloves).

CSIC and Carl Zeiss, Spain sponsored a research fellowship for $\mathrm{Mr}$. Barbero. Ministerio de Educación y Cultura, Spain, provided a research fellowship for Dr. Moreno-Barriuso. This research was funded by grants TIC98-0925-C02-01 (Ministerio de Educación y Cultura, Spain) and CAM: 08.7/0010/2000 (Comunidad Autónoma de Madrid, Spain). Carl Zeiss, Spain lent a Mastervue Atlas Corneal Topography System. herein.

The authors have no proprietary interest in the materials presented

The authors acknowledge Ms. Lourdes Llorente for her help with data collection and analysis, and Mr. Raúl Martín for his contributions in the early stages of the study.

Correspondence: Sergio Barbero, Instituto de Óptica, Consejo Superior de Investigaciones Científicas, Serrano 121, 28006, Madrid, Spain. Tel: 34.915616800; Fax: 34.915645557; E-mail: iodb324@io.cfmac.csic.es

Received: June 11, 2001

Accepted: October 27, 2001
CONCLUSIONS: The similarity found between corneal and total aberration patterns in keratoconus provided a cross-validation of both types of measurements (corneal topography and aberrometry). Both techniques were useful in diagnosing and quantifying optical degradation imposed by keratoconus. [J Refract Surg 2002;18:267-270]

A Ithough the presence of optical imperfections in the eye beyond conventional refractive errors (known as optical aberrations) have been noticed for more than a century ${ }^{1}$, it is only in the last few years that they have been considered from a clinical perspective. Interest has been generated from evaluation of refractive surgery outcomes, and by the increasing possibilities of correcting (through surgery or other means) high order errors. $^{2-4}$ Corneal topography systems are widely used in the clinic, and in particular, corneal aberrations have been measured following refractive surgery, and the results have been correlated to visual performance. ${ }^{5,6}$ However, the optical quality of the human eye is determined by the optical properties of both the cornea and the lens, as well as their relative alignment and the position of the pupil. ${ }^{7}$ For this reason the measurement of total aberrations provides the most complete description of the image forming properties of the eye. Several types of aberrometers ${ }^{8-10}$ have been used to assess ocular aberrations in normal eyes and following refractive surgery. ${ }^{11-13,15}$ Undoubtedly the combination of information provided by corneal topography and aberrometry provides interesting insight into the properties of individual ocular components. ${ }^{14,15}$ However, both techniques rely on different principles. Typical corneal topographers project a Placido disk (a set of concentric rings) onto the anterior surface of the cornea. Corneal elevation maps are obtained from the distortions of the reflected rings. ${ }^{16}$ The aberrations caused by the front surface of the cornea are then computed by theoretical ray 


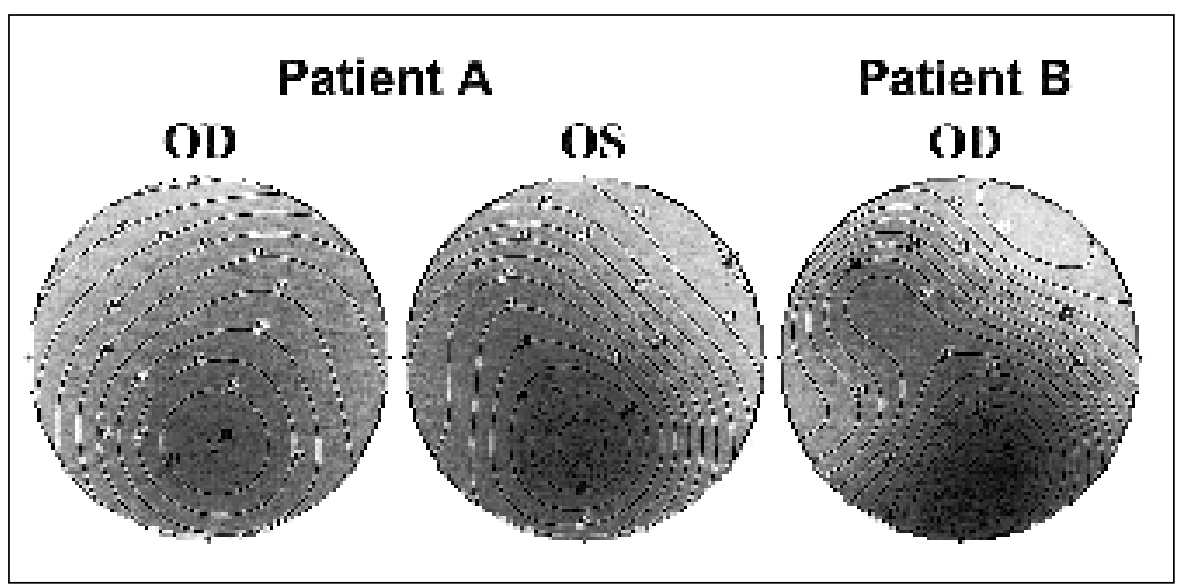

Figure 1. Topographic maps of dioptric power for the three keratoconus eyes in the study. Contour lines are plotted every 1 diopter.

tracing. ${ }^{17,18}$ However, typical aberrometers measure the deviations of beams projected onto the retina through different pupil locations (ie, laser ray tracing ${ }^{19}$, spatially resolved refractometry ${ }^{10}$, or Tscherning's aberroscope ${ }^{20}$ ), or analyze the wavefront as it emerges out of the eye (ie, HartmannShack ${ }^{9}$ ). Factors affecting resolution and accuracy are different across methods (videokeratography and aberrometry). Whereas the wave aberration is computed directly from a set of ray aberrations, the corneal heights are computed from the ring positions and surface location measured from the video images. ${ }^{16}$ Total aberrations are measured directly, whereas some assumptions (ie, index of refraction) are needed to compute corneal aberrations. Prior to comparing total and corneal aberrations, it seems necessary to prove that both techniques are directly comparable. The ideal test are eyes where total and corneal aberrations should be identical, or at least eyes where total aberrations are dominated by the aberrations of the front surface of the cornea. An approximate model of the first case is an aphakic eye. An approximate model of the second is an eye with keratoconus. Given the distinct nature of the two cases, we treat them separately. In this paper, we studied the keratoconus case.

The front surface of the cornea is the major refractive component in the eye, and it is strongly distorted in eyes suffering from keratoconus. ${ }^{21,22}$ Important similarities are therefore to be expected between anterior corneal aberration and total aberration patterns. Furthermore, this comparison can be a good cross-validation of the two techniques used in this study: 1) Computation of anterior corneal aberrations by simulated ray tracing on corneal el evation maps as measured by a Humphrey Atlas corneal videokeratoscope, and 2) laser ray tracing measurements of ocular aberrations. Although presumably relatively small in conven- tional keratoconus, the crystalline lens and posterior corneal surface play a role in overall image quality. ${ }^{14,23}$ This indicates that the measurement of total aberrations may have advantages over the measurement of just anterior corneal aberrations, allowing a better comparison with visual performance. For example, posterior keratoconus, characterized by a conical protrusion of the posterior corneal curvature, a thinned stroma, and non-protruding anterior surface ${ }^{24}$ could be detected measuring the total aberrations, while anterior aberrations would appear as normal. A lack of correspondence between total and anterior corneal aberrations in a diagnosed keratoconus may well be indicative of an involvement of the posterior corneal surface.

\section{PATIENTS AND METHODS}

Total and corneal aberrations were measured on three eyes from two patients: both eyes of patient $A$ (female, age 34) and the right eye of patient $B$ (female, age 40). The three eyes were diagnosed with keratoconus by slit-lamp microscopy, corneal topography, presence of high astigmatism, and reduced visual acuity, being in an early stage for patient $A$ and more advanced in patient $B$. Videokeratography (Humphrey-Zeiss Mastervue Atlas Corneal Topography system, San Leandro, CA) was used during the experimental sessions. Figure 1 shows topographic power maps, revealing corneal inferior steepening in all eyes within ranges reported in the literature as indicative of keratoconus. ${ }^{21,25,26}$ Patient A's autorefractometer refraction was $-2.50-2.50 \times 35^{\circ}$ (OD) and $-2.00-2.00 \times 125^{\circ}$ (OS). Best spectacle-corrected visual acuity (BSCVA) was 20/50 OD and 20/40 OS. Patient B's refraction was $-5.25-5.25 \times 33^{\circ}(\mathrm{OD})$, with a BSCVA of $20 / 100$. The two types of measurements (corneal and total aberrations) were performed in the same experimental session, after recent clinical 
Estimation of Corneal Aberrations From Videokeratography/Barbero et al

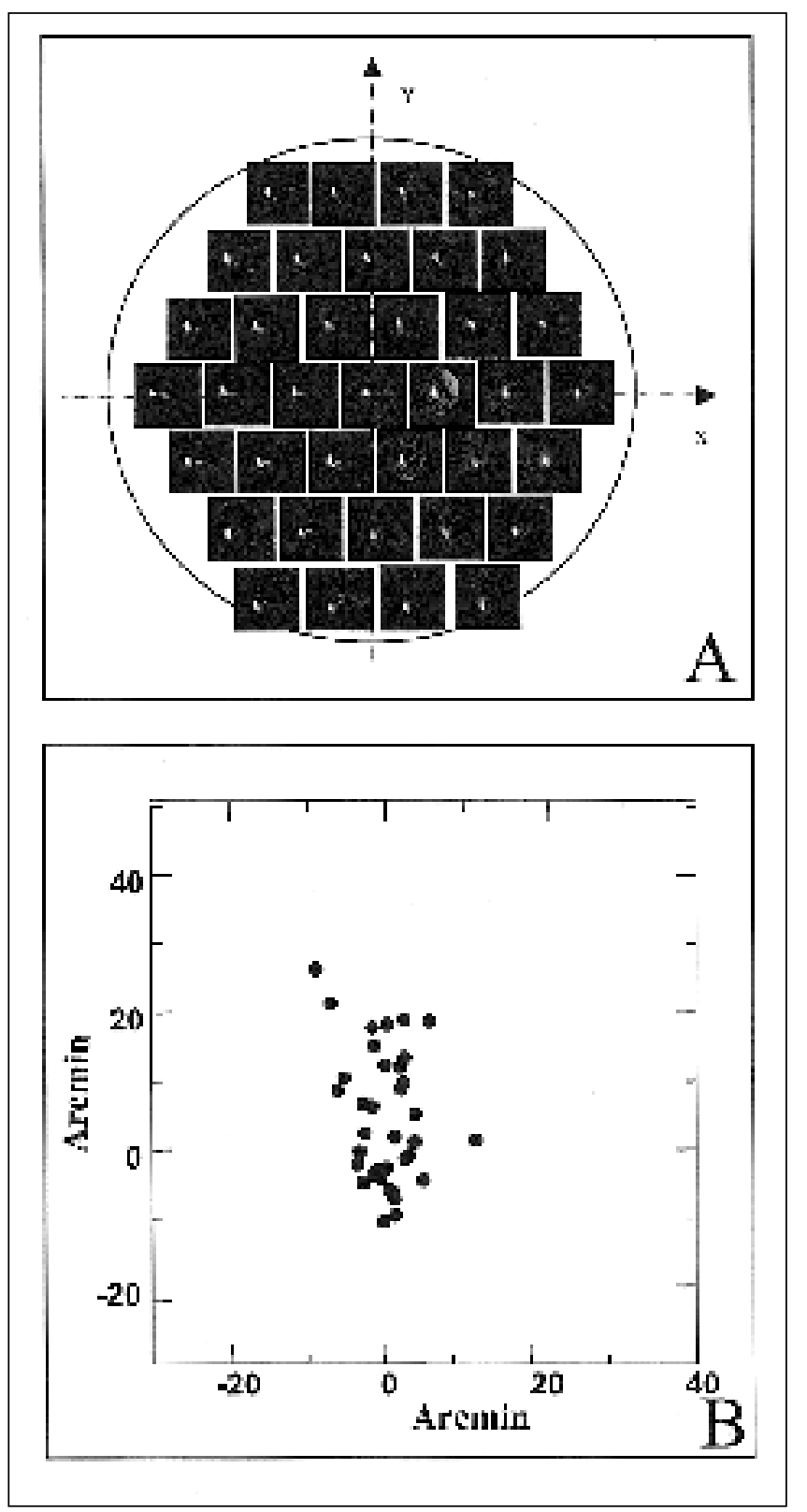

Figure 2. A) Set of retinal images, captured by the high resolution CCD in laser ray tracing as a function of entry pupil location, for patient A, OS. Each retinal aerial image is located at the corresponding entry pupil location. Pupil effective diameter was $6.51 \mathrm{~mm}$. B) Spot diagram, ie, joint plot of the angular ray deviation (in arcmin) from the principal ray (ray passing through the pupil center). These deviations are calculated from the centroids of the retinal images shown in $A$.

screening. Pupils were dilated with one drop of tropicamide $1 \%$. The patients signed informed consent forms approved by institutional ethical committees.

Total aberrations were measured using a laser ray tracing technique. This method has been described in detail. ${ }^{8-11}$ In brief, a set of 37 parallel laser pencils (543 nm HeNe laser) sample the pupil sequentially following a hexagonal pattern. A full scan takes $\sim 4$ seconds. This technique has had similar results and variability as other techniques such as Shack-Hartman and the Spatially Resolved Refractometer. ${ }^{27}$ Simultaneously, the corresponding retinal images are projected onto a high-resolution CCD camera. Figure 2A shows the set of retinal images for one of the runs recorded in a keratoconus eye (Patient A, OS). The location indicates the corresponding entry pupil position. Measurements were done over a 6.51-mm effective pupil diameter for patient A (step-size $=1 \mathrm{~mm}$ ) and $5.5 \mathrm{~mm}$ for patient $B$ (step-size $=0.8 \mathrm{~mm}$ ). We had to reduce slightly the sampled area due to the large amount of aberrations present in patient $\mathrm{B}$. Even with best spherical and cylindrical correction, the aerial images for the most eccentric locations of the 6.51-mm pupil did not fit in the CCD array. Figure $2 \mathrm{~B}$ shows a joint plot of the centroids of those images (spot diagram). The deviation of each centroid from the principal ray (or centroid of the retinal image of the ray passing through the pupil center) is proportional to derivate (slope) of the wave aberration at the corresponding pupil location. This set of values was fit to the derivatives of a 7th order Zernike expansion (35 Zernike coefficients).

A session consisted of five runs (37 images each). All the measurements were foveal. The pupil was continuously monitored with an IR system, and the subjects were stabilized by means of a forehead rest and dental impression, to ensure proper centration and to facilitate positioning reproducibility. Corneal elevation maps were obtained from each eye, using the Mastervue Corneal Topography System. Except for initial control experiments, only one map was captured per eye. The patient fixated foveally, and stabilization was achieved by chin and forehead rests.

The output text files included axial and radial positions, height and curvature data, all obtained from videokeratography images by an arc step reconstruction method. ${ }^{16}$ These data were processed in Matlab (Mathworks, Natik, MA). Points outside a 10-mm region were rejected, since they were usually covered by eyelids or subject to distortion. Polar coordinates were transformed into cartesian coordinates, and the data were interpolated to achieve a rectangular sampling. The corneal height surface was fit to a 7th order Zernike polynomial expansion. ${ }^{28}$ Figure $3 \mathrm{~A}$ shows a typical corneal elevation map in a keratoconic eye (Patient A, OS). I $n$ order to show the irregularities, we subtracted the first six 


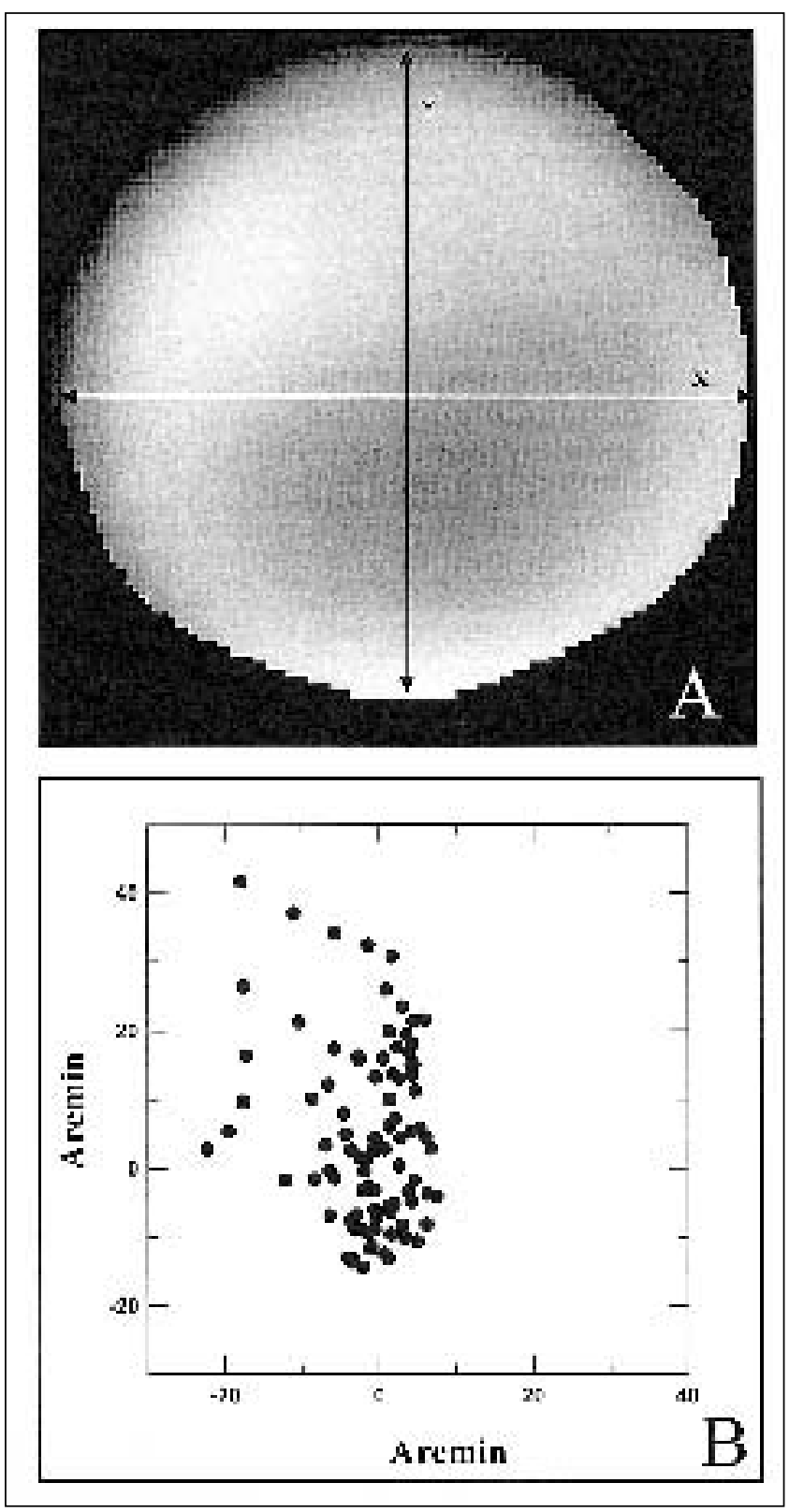

Figure 3. A) Residual height map of patient A, OS. A Zernike polynomial fitting (up to the 2 nd order) has been substracted from raw height data in order to enhance relevant features. ${ }^{29}$ B) Spot diagram obtained by virtual ray tracing on corneal height data (Patient $A$, OS). Stop pupil diameter was $6.51 \mathrm{~mm}$ after appropriate centration.

terms of Zernike polynomial fit to the height data from the raw height data. ${ }^{29} \mathrm{~F}$ irst derivatives in $\mathrm{x}$ and $y$, and second cross derivatives in $x y$ from the corneal surface were also computed. These data are the input for the optical design program, Zemax V.9 (F ocus Software, Tucson, AZ), used to perform a virtual ray tracing simulation. We simulated a set of parallel light pencils $(\lambda=543 \mathrm{~nm})$ coming from infinity, sampling $64 \times 64$ points of the corneal surface (in a rectangular grid). The indices of refraction were taken as that of the air and the aqueous humor (1.3391). Figure 3B shows the simulated spot diagram for the example shown in Figure 3A. The corneal wave aberration was described as a 7th order Zernike polynomial expansion and evaluated at the plane of best focus. Unlike the laser ray tracing measurements (where the reference was the pupil center), corneal topography typically uses the corneal reflex for alignment. The system does not allow acquisition of out-of-focus images, so images with centration errors of more than about $0.25 \mathrm{~mm}$ were not captured. Proper alignment of corneal and total wave aberration is necessary for direct comparison. ${ }^{30}$ To compensate for possible shifts in the reference axis, we developed custom software to locate the pupil position that produced minimum difference of corneal to total aberrations. Corneal aberrations were computed over a large pupil diameter $(10 \mathrm{~mm})$ and re-computed over a smaller pupil (matching the pupil size of total aberration measurements), moving the center over a 1-mm square region around the position of the corneal reflex, at 0.1-mm steps. A difference total-corneal map was computed for each pupil location. This surface showed a minimum, typically slightly decentered from the corneal reflex. Independent observations of the corneal reflex relative to the pupil center ${ }^{31}$ performed on control subjects show that this procedure identifies the pupil center (inaccessible otherwise from the corneal topography images).

Even if the decentration between the corneal reflex and pupil center are corrected, there is still a tilt between the keratometric axis, used in videokeratography, and the line of sight, used in laser ray tracing. This angle can be computed from the distance between the corneal intersect of the keratometric axis and corneal sighting center (intersection of line of sight with anterior corneal surface) and the distance from the fixation point to front corneal surface, $148.3 \mathrm{~mm}$ in our videokeratoscope (Steve Kaatmann, Zeiss Humphrey Systems, personal communication). As the position of corneal sighting center was not available in our patients, we assumed the average value reported by Mandell and colleagues $^{32}(0.38 \pm 0.10 \mathrm{~mm}$, across 20 normal eyes). With these values, we computed a corneal tilt of $-0.15^{\circ}$, which we neglected in further computations. For the eye of patient B we found that, considering this average tilt, root mean square changed only by $2.6 \%$ (aphakic eye) for 3rd order terms, and $0.6 \%$ for spherical aberration. 


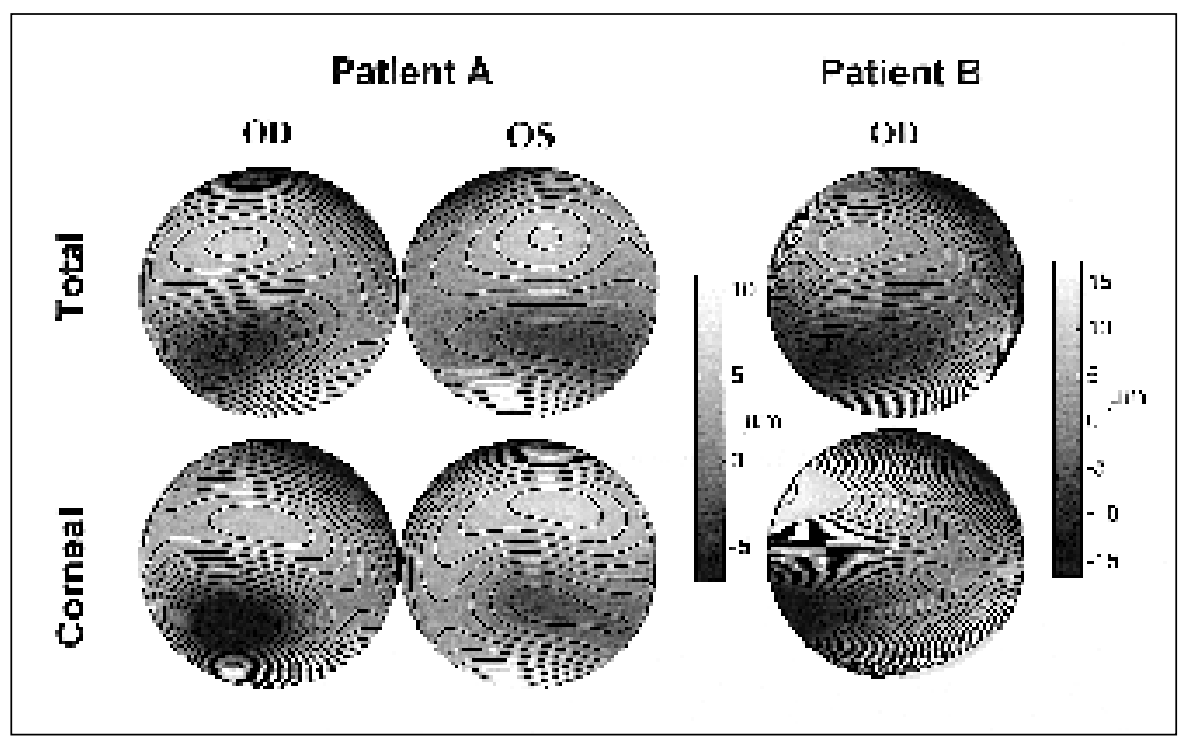

Figure 4. Wave aberration patterns (without tilts and defocus) in the three measured eyes, for total aberrations (upper row) and corneal aberration (lower row). Contour lines are plotted every $1 \mu \mathrm{m}$. The gray scale pattern represents wave aberration heights in microns. Diameters were $6.51 \mathrm{~mm}$ in patient $A$ and $5.51 \mathrm{~mm}$ in patient $\mathrm{B}$.

We analyzed individual Zernike terms to compare the corneal and total aberrations. Root mean square wavefront error (RMS) was used as an optical quality metric.

\section{RESULTS}

Figure 4 shows total (upper row) and corneal (lower row) wave aberration maps for patients $A$ (OD and OS) and patient $B$ (OD). Contours have been plotted at $1-\mu \mathrm{m}$ intervals. Pupil sizes are $6.51 \mathrm{~mm}$ for patient $A$ and $5.5 \mathrm{~mm}$ for patient $B$. The gray scale for corneal and total aberrations is the same for each patient. Tilt and defocus have been cancelled in all eyes. There is good correspondence between corneal and total wavefront maps. Peak-tovalley values are double in patient $B$ than in patient $A$.

Figure 5 compares corneal (open diamonds) and total (solid circles) Zernike coefficients for each eye, following the ordering and notation recommended by the Optical Society of America Standard Committee. ${ }^{33}$ For patient $A$, there is good correspondence between total and corneal aberrations. In both eyes of this patient, the dominant aberration is the coma term $Z_{3}{ }^{-1}$, which is higher than astigmatism. The dominance of coma is also evident in the wave aberration plots.

The largest difference between total and corneal aberration for patient $A$ was found for spherical aberration (coefficient of $Z_{4}{ }^{0}$ element). $F$ or the right eye, corneal and total spherical aberration have a different sign and show a total difference of $0.74 \mu \mathrm{m}$, whereas for the left eye corneal spherical aberration exceeds total spherical aberration by $0.55 \mu \mathrm{m}$. This indicates a compensation of corneal spherical aber- ration by spherical aberration of the crystalline lens. This balance of spherical aberration is a common finding in normal eyes. ${ }^{14,34,35}$ Although the progressive disease seems to affect high order terms (particularly coma) of both corneal and total aberrations, it does not seem to modify the amount of spherical aberration $\left(Z_{4}{ }^{0}\right)$.

In patient $B$, the correspondence between corneal and total Zernike terms is worse than for patient $A$. Some exceptional terms show large differences. Major differences are found in astigmatic term $\mathrm{Z}_{2}^{-2}$ (4.58- $\mu \mathrm{m}$ difference), third order term $Z_{3}^{3}(0.63 \mathrm{~mm})$ and 4th order term $Z_{4}^{-4}(1.15 \mu \mathrm{m})$. In this subject, astigmatism is the dominant term, followed by coma.

For the sake of clarity, error bars have not been plotted in Figure 5. Control experiments performed in one patient show a mean standard deviation of $0.08 \mu \mathrm{m}$ for the corneal Zernike coefficients (averaged across terms, excluding tilts and defocus). The mean standard deviation for the total Zernike coefficients (averaging across the three eyes and coefficients) was $0.13 \mu \mathrm{m}$.

The Table shows the RMS for different terms and orders evaluated for the three eyes. There is a clear predominance of 3rd order (coma-like) terms, both in corneal and total aberration. In terms of variance (squared RMS), they represent $61 \%$ (70.72\% for patient A and $41.53 \%$ for patient B) of the aberration (excluding tilt and defocus, but including astigmatism). Excluding astigmatism, coma-like terms represent $90.85 \%$ of the variance. Mean 3rd order aberration $(2.02 \pm 0.41)$ in this group of keratoconus eyes exceeds by a factor of 3.74 the average $3 r d$ order aberration $(0.54 \pm 0.30)$ of a group of normal 
eyes. This control group of 22 eyes from 12 subjects was within similar age range $(28 \pm 5 \mathrm{yr})$ and within similar refractive errors $\left(-6.42 \pm 2.50 \mathrm{D}\right.$ sphere). ${ }^{11}$

\section{DISCUSSION}

Corneal and total aberrations were estimated in three eyes, all diagnosed with keratoconus at different stages of disease. We found good correspondence between corneal and total aberrations, particularly in both eyes of patient $A$, indicating that the overall aberration pattern is dominated by the front corneal surface, and that both methods are able to capture similarly the distortions produced by the irregular cornea. Our results show that the Humphrey Mastervue Atlas corneal topography system and laser ray tracing are both adequate tools to analyze optical quality in keratoconus. As reported ${ }^{36}$, image degradation in keratoconus is mainly due to an increase in higher order aberrations, particularly coma. In the three affected eyes from this study, third order aberrations increased by a factor of 4.24 , 2.87 , and 4.13 , respectively, with respect to normal eyes. ${ }^{11}$

In these eyes with abnormally high order aberrations, both techniques provided good results (valid topography data and good quality retinal images in laser ray tracing). Both techniques failed in two eyes with a highly advanced stage of keratoconus (one patient scheduled for keratoplasty, not shown here). In these eyes, the videokeratographic images were so distorted that the commercial software did not accept the data. Many of the laser ray tracing aerial retinal images were highly diffused (probably due to corneal scarring), and even after compensation of spherical error they did not fit within the CCD area. There are many differences inherent to the techniques under use, nevertheless the similarity of the corneal and total aberration pattern is high, at least for patient $A$. The accuracy of the measurements is determined by different factors. The fact that despite these differences the results are similar indicate that these factors do not seem to be essential. Studies of the accuracy of corneal topography devices show that they can measure to a root mean square error of $3.7 \pm 0.7 \mu \mathrm{m}$, at least regular surfaces (5.4-mm radius aspheric surfaces). ${ }^{37}$ It may be argued that errors may arise from smooth Zernike fitting to the corneal heights.

To test the limitations of the polynomial smoothing of the surface in the patients in this study, we fit the corneal heights to Zernike polynomials of increasing order (5th through 10th order) and computed the corresponding root-mean-square fitting

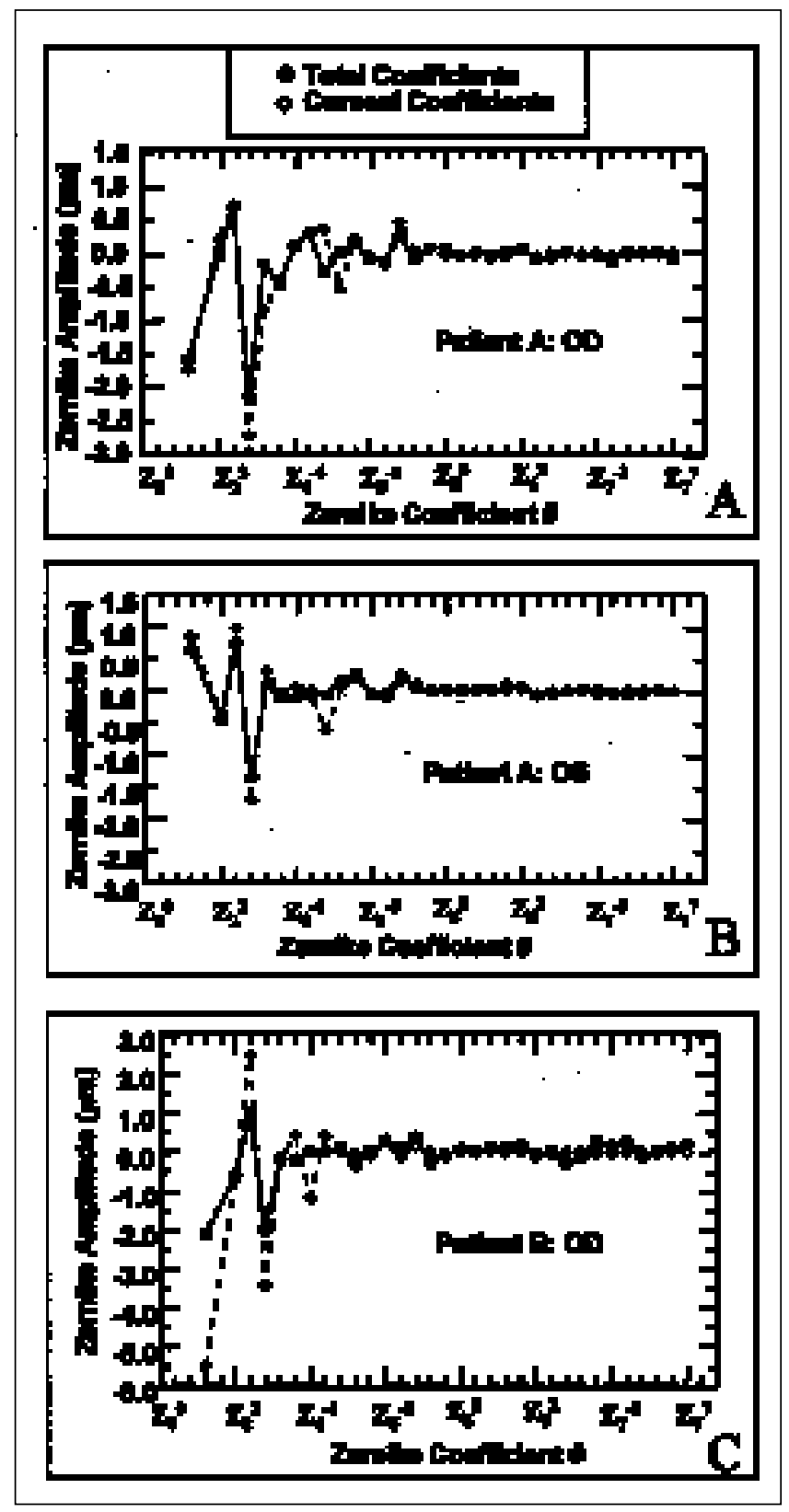

Figure 5. Total (solid circles) and corneal (empty diamonds) aberrations for A) Patient A, OD, B) Patient A, OS, and C) Patient B, OD. Notation follows the OSA Standard Committee's recommendations. ${ }^{33}$

errors. We observed that little further improvement was found using expansions of orders higher than 6 th. This agrees with previous studies that showed that the optimal number of Zernike terms to fit the corneal surface depends on the actual corneal shape, and it is not necessarily the highest order expansion. ${ }^{38}$ We fit the corneal surface to a 7th order polynomial expansion. For patient $A$, the root mean square fitting error was $0.95 \mu \mathrm{m}$ OD and $0.74 \mu \mathrm{m}$ 


\begin{tabular}{|c|c|c|c|c|c|c|}
\hline \multicolumn{7}{|c|}{$\begin{array}{c}\text { Table } \\
\text { Root Mean Square (RMS) and Zernike Corneal and Total Terms ( } \mu \mathrm{m})\end{array}$} \\
\hline & \multicolumn{2}{|c|}{ Patient A (OD) } & \multicolumn{2}{|c|}{ Patient A (OS) } & \multicolumn{2}{|c|}{ Patient B (OD) } \\
\hline & Total & Corneal & Total & Corneal & Total & Corneal \\
\hline$\overline{Z_{2}^{-2}}$ & 1.58 & 1.73 & -0.64 & -0.85 & -2.1 & -5.24 \\
\hline $\mathrm{Z}_{2}^{+2}$ & 0.01 & -0.22 & 0.44 & 0.34 & -0.6 & -0.66 \\
\hline RMS 2nd to 7th order (except defocus) & 2.84 & 3.55 & 1.8 & 2.3 & 3.25 & 7.1 \\
\hline RMS 3rd order & 2.29 & 2.95 & 1.55 & 1.99 & 2.23 & 4.26 \\
\hline RMS 3rd and higher orders & 2.36 & 3.09 & 1.62 & 2.11 & 2.4 & 4.46 \\
\hline RMS 4th order & 0.46 & 0.77 & 0.35 & 0.66 & 0.33 & 1.24 \\
\hline RMS 5th and higher orders & 0.38 & 0.52 & 0.28 & 0.27 & 0.82 & 0.38 \\
\hline \multicolumn{7}{|l|}{ Variance* $3 r d * 100$} \\
\hline Variance 2nd and higher (no defocus) & 64.9 & 69.1 & 74.1 & 74.9 & 47.1 & 36 \\
\hline Variance $3 r d * 100$ & & & & & & \\
\hline Variance 3rd and higher & 94.1 & 91.1 & 91.6 & 89 & 86.3 & 93 \\
\hline
\end{tabular}

OS. F or patient $B$, the fitting error $(3.76 \mu \mathrm{m})$ was of the same order than the nominal accuracy of the device. Increasing the Zernike expansion up to the 10th order only reduced this error to $3.52 \mu \mathrm{m}$. This finding suggests that this amount of aberrations may set a limit where a Zernike polynomial expansion to the surface can be a good approximation.

For patient A, accuracy of corneal aberration computation is clearly limited by the topographer, not by the fitting algorithm. To evaluate the influence of topography measurement accuracy on corneal aberration errors, we simulated a corneal surface with data randomly separated from the original topography data (patient A, left eye) by a mean value of $3.7 \mu \mathrm{m}$. We obtained a wavefront average error of $0.005 \mu \mathrm{m}$. This wavefront error is lower than run-to-run variability and total wave aberration errors. The Zernike polynomial fit to the 7th order seems to be an appropriate description of wavefront data (both corneal and total). Sampling density in the ray tracing simulation was 4096, but we also tried denser sampling (16384 and 65536) and the differences that we found were negligible. A much sparser sampling (37) was used in laser ray tracing experimental measurements of total aberrations, and nevertheless we found consistent results. This suggests that the major aberrations present in keratoconus do not require a dense sampling to be captured.

Our centration algorithm allows direct comparison of corneal and total aberration maps, both centered with respect to the pupil center. Previous studies $^{32}$ found a mean distance (in 20 eyes) between corneal sighting center (pupil center in the cornea) and videokeratography map center of $0.38 \pm$ $0.1 \mathrm{~mm}$. For the eyes shown in this study, we found a mean distance between corneal reflex and pupil center of $0.5 \pm 0.1 \mathrm{~mm}$, and $0.6 \pm 0.5 \mathrm{~mm}$ for a larger population of 89 eyes on which we used the same procedure. These slightly higher differences could be due to the fact that dilated eyes increase pupil center shift by about $0.2 \mathrm{~mm}$ on average. ${ }^{39,40}$

In summary, we have crossed-validated two techniques for measuring corneal and total aberrations with tests on eyes with keratoconus. They have proved powerful to detect and quantify the aberrations in moderate keratoconus. The data on patient $B$ probably sets a limit where the assumptions of the techniques are valid to provide valid quantitative data. Zernike polynomial corneal height fitting error equals the accuracy of the corneal topography device, and we had to use a smaller pupil diameter in the laser ray tracing system to capture the entire set of retinal images. Measurements in a very advanced keratoconus failed with both instruments. Finally, part of the differences found between specific terms of corneal and total aberrations in patient B might have been caused by the posterior corneal surface being affected in advanced keratoconus. ${ }^{22}$ In this regard, measurement of overall aberrations has advantages over corneal topography, since it allows capturing possible alterations of the posterior corneal surface. Since they contain information of all optical components (including the crystalline lens) they provide the most complete description of the imaging properties of the eye. 


\section{REFERENCES}

1. Helmholtz HV. Physiological Optics. New York, NY: J .P.C. Southhall; 1896.

2. MacRae SM, Schwiegerling J , Snyder R. Customized corneal ablation and super vision. J Refract Surg 2000;16(suppl): S230-S235.

3. LiangJ , Williams D, Miller D. Supernormal vision and highresolution retinal imaging through adaptive optics. J Opt Soc Am 1997; 14:2884-2892.

4. Navarro R, Moreno E, Bará S, Mancebo T. Phase-plates for wave-aberration compensation in the human eye. Opt Lett 2000;25:236-238.

5. Marcos S. Aberrations and visual performance following standard laser vision correction. J Refract Surg 2001;18:596-601.

6. Applegate RA, Hilmantel G, Howland H, Tu EY, Starck T, Zayak J. Corneal first surface optical aberrations and visual performance. J Refract Surg 2000;16:507-514

7. Marcos S, Burns SA, Prieto PM, Navarro R, Baraibar B. Investigating the sources of monochromatic aberrations and transverse chromatic aberration. Vision Res, in press.

8. Navarro R, Losada MA. Aberrations and relative efficiency of light pencils in the living human eye. Optom Vis Sci 1997; 74:540-547.

9. Liang J , Grimm B, Golez S, Bille J . Objective measurement of wave aberrations of the human eye with the use of a Hartmann-Shack wave-front sensor. J Opt Soc Am A 1994;11:1949-1957.

10. He J C, Marcos S, Webb RH, Burns SA. Measurement of the wave-front aberration of the eye by a fast psychophysical procedure. J Opt Soc Am A 1998;15:2449-2456.

11. Moreno-Barriuso E, Merayo-Lloves J , Marcos S, Navarro R, Llorente L, Barbero S. Ocular aberrations before and after corneal refractive surgery: LASIK-induced changes measured with laser ray tracing. Invest Ophthalmol Vis Sci 2001:42:1396-1403.

12. Thibos LN, Hong $X$. Clinical applications of the ShackHartmann aberrometer. Optom Vis Sci 1999;76:817-825.

13. Seiler T, Kaemmerer M, Mierdel P, Krinke H-E. Ocular optical aberrations after photor efractive keratectomy for myopia and myopic astigmatism. Arch Ophthalmol 2000;118:17-21.

14. Artal P, Guirao A. Contribution of the cornea and the lens to the aberrations of the human eyes. Opt Lett 1998;23: 1713-1715.

15. Marcos S, Barbero S, Llorente L, Merayo-Lloves J. Optical response to myopic LASIK surgery from total and corneal aberration measurements. Invest Ophthalmol Vis Sci 2001;42:3349-3356.

16. Campbell C. Reconstruction of the corneal shape with the MasterVue Corneal Topography System. Optom Vis Sci 1997;74:899-905.

17. Camp ] J, Maguire LJ , Cameron BM, Robb RA. A computer model for the evaluation of the effect of corneal topography on optical performance. Am J Ophthalmol 1990;109:379386.

18. Sarver EJ, Applegate RA. Modeling and predicting visual outcomes with VOL-3D. J Refract Surg 2000;16(suppl): S611-S116.

19. Navarro R, Moreno-Barriuso E. A laser ray tracing method for optical testing. Opt Lett 1999;24:951-953.

20. Mrochen $M$, Kaemmerer M, Mierdel P, Krinke HE, Seiler T. Principles of Tscherning aberrometry. J Refract Surg 2000;16(suppl):S570-S571.

21. Maguire LJ , Bourne WM. Corneal topography of early keratoconus. Am J Ophthalmol 1989;108:107-112.

22. Tomidokoro A, Oshika T, Amano S, Higaki S, Maeda N, Miyata K. Changes in anterior and posterior corneal curvatures in keratoconus. Ophthalmology 2000;107:1328-1332.

23. Smith G, Cox MJ , Calver R, Garner LF. The spherical aberration of the crystalline lens of the human eye. Vision Res 2001;41:235-243.
24. Mannis MJ , Lightman J , Plotnik RD. Corneal topography of posterior keratoconus. Cornea 1992;11:351-354.

25. Mohammad H, Hashemi $\mathrm{H}$. A quantitative corneal topography index for detection of keratoconus. J Refract Surg 1998;14:427-436.

26. Maeda N, Klyce SD, Smolek MK, Thompson HW. Automated keratoconus screening with corneal topography analysis. Invest Ophthalmol Vis Sci 1994;35:2749-2757.

27. Moreno-Barriuso E, Marcos S, Navarro R, Burns S. Comparing laser ray tracing, spatially resolved refractometer, and Hartmann-Shack sensor to measure the ocular wave aberration. Optom Vis Sci 2001;78:152-156.

28. Schwiegerling J, Greivenkamp JE, Miller J M. Representation of videokeratoscopic height data with Zernike polynomials. J Opt Soc Am A 1995;12:2105-2113.

29. Schwiegerling J. Cone Dimensions in Keratoconus using Zernike polynomials. Optom Vis Sci 1997;74:963-969.

30. Applegate RA, Thibos LN, Bradley A, Marcos S, Roorda A, Salmon TO, Atchison DA. Reference axis selection: Subcommitte report of the OSA working group to establish standards for measurement and reporting of optical aberrations of the eye. J Refract Surg 2000;16:S656-S658.

31. Marcos S, Burns SA. On the symmetry between eyes of wavefront aberration and cone directionality. Vision Res 2000;40:2437-2447.

32. Mandell RB, Chiang CS, Klein SA. Location of the major corneal reference points. Optom Vis Sci 1995;72:776-784.

33. Thibos LN, Applegate RA, Schwiegerling JT, Webb R, Members VST. Standards for reporting the optical aberrations of eyes. Vision Science and its Applications. Tops Volume 2000;35.

34. EI Hage SG, Berny F. Contribution of the crystalline lens to the spherical aberration of the eye. J Opt Soc Am 1973;63:205-211.

35. Sivak J G, Kreuzer RO. Spherical aberration of the crystalline lens. Vis Res 1983;23:59-70.

36. Lagana MA, Cox IG, Potvin RJ. The effect of keratoconus on the wavefront aberration of the human eye. Invest Ophthalmol Vis Sci 2000;41(suppl):S679.

37. Schultze RL. Accuracy of corneal elevation with four corneal topography systems. J Refract Surg 1998;14:100-104.

38. Iskander DR, Collins MJ, Davis B. Optimal modeling of corneal surfaces with zernike polynomials. IEEE Trans Biomed Eng 2001;48:87-95.

39. Uozato H, Guyton DL. Centering corneal surgical procedures. Am J Ophthalmol 1987;103:264-275.

40. Walsh $\mathrm{G}$. The effect of mydriasis on the pupillary centration of the human eye. Ophthalmic Physiol Opt 1988;8:178-182.

\section{Author:}

Fig 3A, what do the shadings mean since there is no calibration given. 